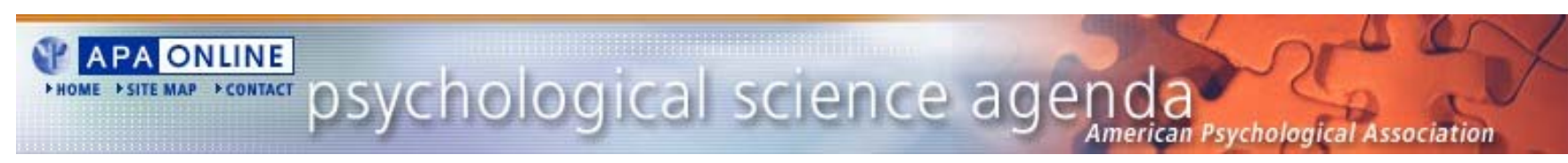

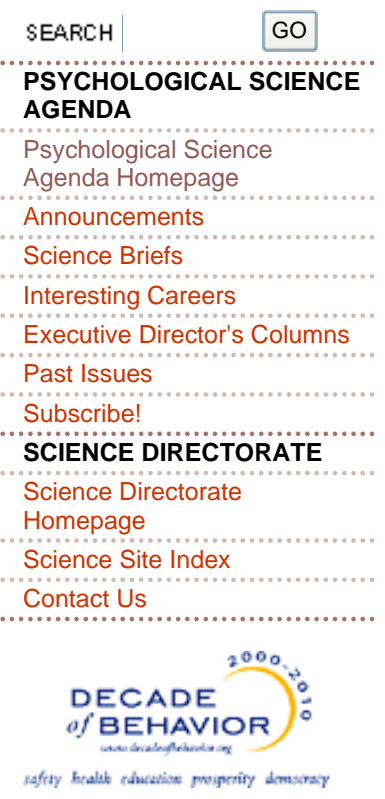

Volume 22: No. 5, May 2008

Vol No. 5 , May 2008

\title{
Brain Bases of Individual Differences in Cognition
}

by Chantel S. Prat and Marcel Adam Just, Carnegie Mellon University

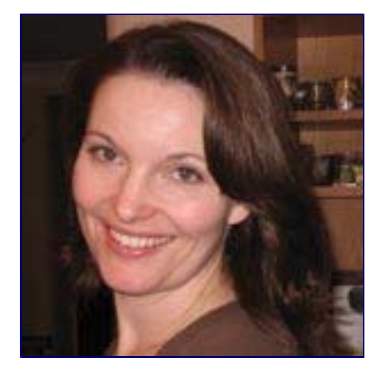

Chantel Prat is a postdoctoral fellow at the Center for Cognitive Brain Imaging at Carnegie Mellon University. She earned her Ph.D. at the University of California, Davis, working with Debra Long on investigations of individual differences in discourse representation across the two hemispheres. She is currently using fMRI to explore the brain basis of individual differences in language and cognition. Her research emphasizes network-level characterizations of brain function with a special interest in how the role of the right hemisphere in language processes varies across individuals and tasks.

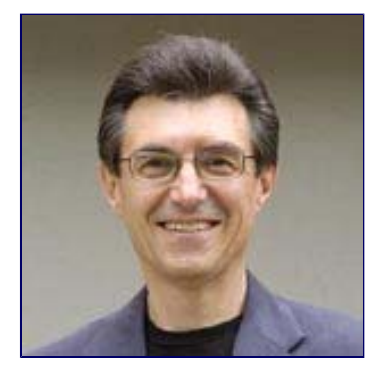

Marcel Adam Just is the D. O. Hebb Professor of Psychology and the Director of the Center for Cognitive Brain Imaging at Carnegie Mellon. $\mathrm{He}$ is the author of theoretical and empirical articles on language processing, reasoning, visuo-spatial reasoning, individual differences, and autism. The research uses brain imaging (fMRI) to study the neural basis of the architecture of cognition. The fMRI findings are being used in the development of a computational theory of cognition that is based on the dynamic, collaborative activity of the relevant cortical components, each drawing on its own set of relative specializations. The theory provides a mapping between cognitive function and brain activity.

Individual differences in the ability to execute cognitive processes must ultimately be underpinned by differences in neural functioning. Over the past decade, research on the relation between higher-level cognition and brain function has characterized thinking as the functioning of a large-scale cortical network, schematically depicted in Figure 1 as a set of cortical centers connected by communications pathways. Moreover, individual differences in this cortical system, which in essence is a biological thinking machine, are likely to be dependent on the availability and efficient deployment of finite neural resources (Just \& Varma, 2007). Given this perspective, our explorations of the brain basis of individual differences in cognition have focused on network-level characteristics of brain function and on resource availability and deployment. We have proposed that a set of at least three cortical network-level attributes 
underlie individual differences in cognitive performance ${ }^{1}$ (Newman \& Just, 2005; Prat, Keller, \& Just, 2007), namely: 1 . efficient use of neural resources, 2. high synchronization (coordination) between cortical centers, and 3. adaptation of cortical networks in the face of changing demands. Below, we outline methods for quantifying neural efficiency synchronization, and adaptation, and describe how they relate to individual differences in cognition in neurotypical adults $^{2}$. We also propose potential brain-behavior links for some mental processing attributes (speed and fluid intelligence) in which individuals may vary.

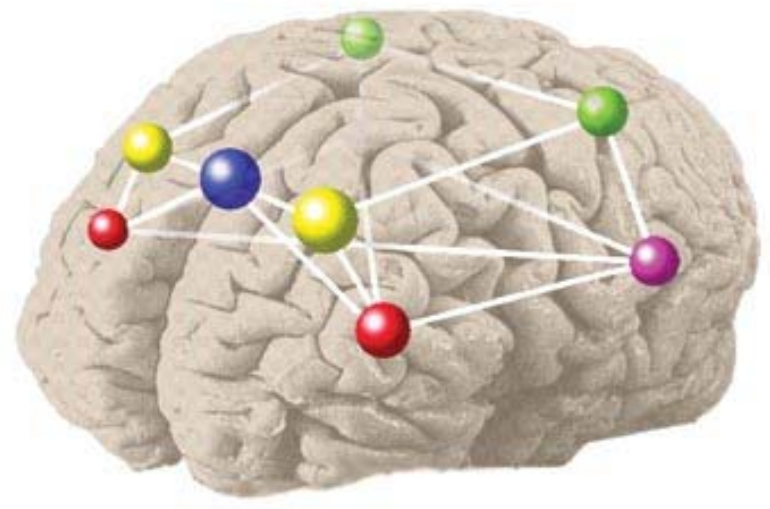

Figure 1. Schematic depiction of a cortical network.

Neural Efficiency. Individual differences in cognitive functioning are likely to be reflected by the amount of mental resource consumption required to effectively perform a task. Resource consumption can be measured by the amount of brain activation observed in a given cortical center during task performance, such that less activation at a given level of proficiency and a given amount of practice implies higher neural efficiency. Of the three brain attributes we discuss, neural efficiency was the first to be associated with individual differences, long before the days of brain imaging. For example, an early electrophysiological investigation of good and poor readers provided evidence that good readers had more efficient neural processes (as indexed by lower power spectra in EEG) than did poor readers (Maxwell, Fenwich, Fenton, \& Dollimore, 1974). More recently, both PET and fMRI investigations have generally found that individuals with higher skill levels tend to utilize fewer neural resources (generate less brain activity, all other things being equal) than individuals with lower skill levels (e.g., Boivin et al., 1992; Haier et al., 1988; Newman, Carpenter, Varma \& Just, 2003; Reichle, Carpenter, \& Just, 2000).

One neuroimaging investigation of individual differences showed that the match between an individual's cognitive abilities and the resources necessary to perform a task are important for efficiency considerations. Using a sentence-picture verification paradigm, Reichle and colleagues manipulated the use of either verbal or visual strategies in individuals with varying verbal and visual-spatial skill (Reichle et al., 2000). They found that participants with higher verbal abilities, as measured by reading span, had lower activation volumes in typical language regions (e.g., Broca's area) when engaging in verbal strategies. Similarly, individuals with higher visual-spatial skills, as measured by mental rotation ability, had lower activation volumes in typical visual association regions (e.g., parietal cortex) when engaging in spatial strategies. Thus, individuals with greater ability in a certain domain showed more efficient neural processing in the cortical regions that supported that domain, when using a strategy that evoked that specific type of processing.

In language processing, the extent to which the right hemisphere $(\mathrm{RH})$ contributes to performance on a task may be a function of neural efficiency. Specifically, in a less-skilled individual who requires more neural resources to perform a task, neural processing may "spill over" from the traditional left hemisphere (LH) language regions into $\mathrm{RH}$ homologues. Support for this spillover hypothesis of $\mathrm{RH}$ language function has been found in several studies that show that less-skilled readers have greater RH participation in language tasks than do skilled readers. In a series of divided visual field experiments investigating discourse representation, less-skilled readers showed priming in both the $\mathrm{LH}$ and $\mathrm{RH}$ for a series of discourse relations, whereas skilled readers showed only LH priming for the same relations (Prat, Long, \& Baynes, 2007). More recently, in an fMRI investigation of individual differences in sentence comprehension, greater $\mathrm{RH}$ (as well as $\mathrm{LH}$ ) activation was found in less-skilled participants than in skilled participants (Prat, et al., 2007). The most convincing evidence for RH spillover, however, comes from a recent fMRI investigation of inference comprehension. In a group of 19 readers with varying reading skill levels, reliable negative correlations were observed between neural activation in the $\mathrm{RH}$ and reading skill (Prat, Mason, \& Just, in preparation). To illustrate the relation between reading skill and right hemisphere activation (in right-handed participants), Figure 2a depicts a high degree of RH activation during inference comprehension for the lowest skilled reader in the group, whereas Figure $2 \mathrm{~b}$ depicts a low degree of $\mathrm{RH}$ activation in the highest-skilled reader in the group. 


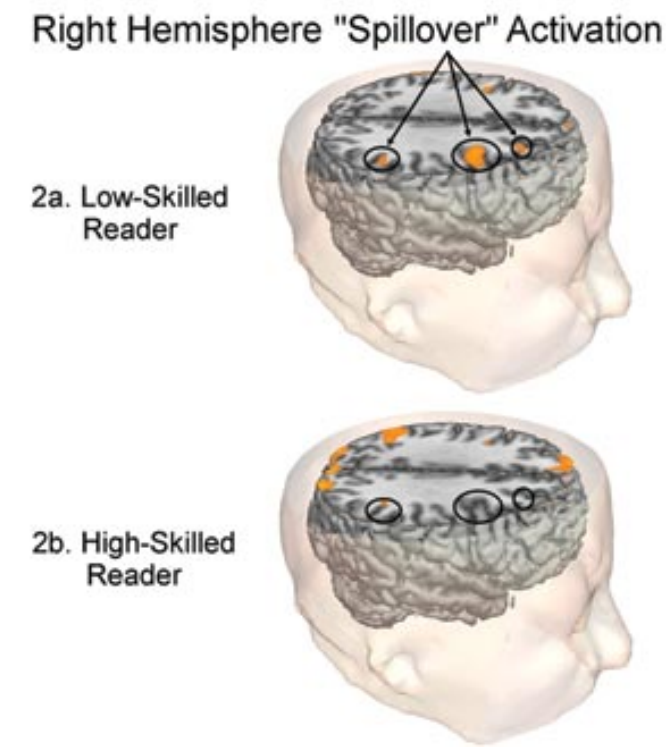

Figure 2. Activation maps depicting RH "spillover" for the lowestskilled reader but not the highest-skilled reader during an inference comprehension task.

In the quantification and comparison of the activation that provides an index of neural efficiency, it is important to ensure that all individuals performing a task are using the same strategy and are performing the task successfully. For example, if individuals with lower skill levels were failing to complete the task or using a less effective high-level strategy (algorithm) and thus showing less activation, this would not constitute an adequate basis for a comparison of neural efficiency. One useful paradigm for studying individual differences in neural efficiency attempts to correlate a psychometric score (e.g., working memory capacity or IQ) with some index of neural efficiency (e.g., number of voxels activated above threshold) while individuals perform a well-specified task that is within the range of abilities of all the participants (e.g., sentence comprehension). When potential confounds such as strategy and accuracy are accounted for, skilled individuals generally exhibit more efficient neural processes, manifested as less activation.

Neural Synchronization. The degree of synchronization between regions of a cortical network is another characteristic of neural functioning that is related to individual differences in performance. It is worth noting that synchronization is a characteristic of the functioning of a distributed cortical network, rather than a characteristic of a particular brain area. To execute complex cognitive processes, the various cortical regions involved in performing a task must be able to effectively communicate and synchronize their individual processes. For example, in a language task, the areas responsible for executing subcomponent processes such as syntactic parsing and semantic integration must collaborate to synthesize the information necessary for comprehension. Such collaboration may be measured in functional neuroimaging studies by the correlation of the activation time series in a given region with the activation time series of another region. The extent to which the activation levels of two regions rise and fall in tandem is taken as a reflection of the degree to which the two regions are functionally connected, and the term that is widely used to refer to the activation time series correlation is functional connectivity (Friston, Jezzard, \& Turner, 1994)

Research on functional connectivity suggests that it is an important index of neural functioning. For example, functional connectivity increases with learning, in a pattern that is very similar to the pattern of improvements in behavioral performance (Buchel, Coull, \& Friston, 1999). There is also systematic variation in functional connectivity that is related to age/performance differences; in a working memory task, younger individuals, who performed better on the task, had higher functional connectivity than did older individuals, who performed poorly on the task (Otsuka \& Osaka, 2005). Our research has shown that functional connectivity is often higher in skilled readers than in less-skilled readers for a number of reading tasks (Prat, et al., 2007; Prat, et al., in preparation). Figure 3 depicts the relation between the functional connectivity (between left and right inferior frontal regions) during inference generation to the level of the participants' reading skill (measured by the Nelson-Denny percentile). 


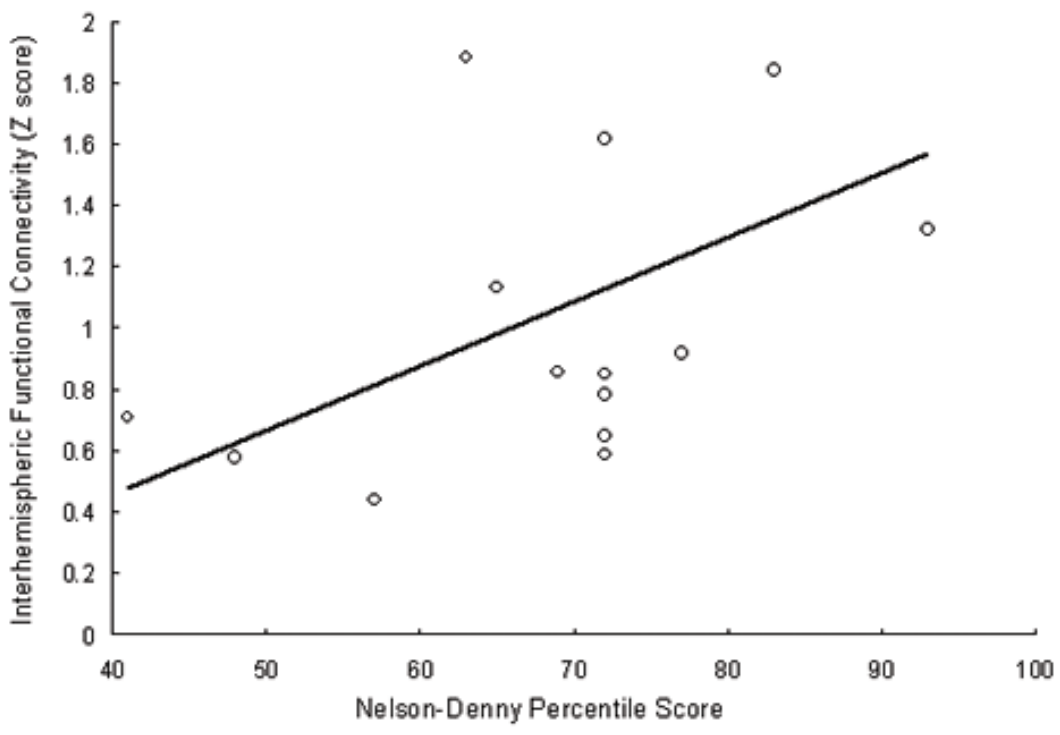

Figure 3. Scatterplot illustrating the increase in synchronization between left and right inferior frontal gyri as a function of increasing reading skill.

In summary, the synchronization between cortical regions provides an index of network coordination, and individual differences in this synchronization are related to individual differences in skill level. In general, higher skill level is associated with higher levels of cortical synchronization.

Neural Adaptability. The cognitive demands imposed by complex tasks are dynamic in nature, changing quantitatively and qualitatively from second to second. Therefore, an effective cortical system must be able to adapt to meet the changing demands. Adaptability is measured by the change in neural activation patterns as a function of changing task demands. Brain imaging studies provide evidence of such adaptation in terms of the activation of brain areas on an asneeded basis. Although a modal set of areas activates for any given task, additional areas may be recruited to deal with changes in the level or type of demand. For example, if the comprehension of the latter part of a sentence requires problem-solving processes, dorsolateral prefrontal cortex becomes activated at the time that portion of the sentence is being read (Newman, Just, \& Carpenter, 2002).

The adaptive modulation of activity in the face of changing task demands is related to individual differences in performance. For example, one PET investigation of individual differences in reasoning abilities found that individuals with high reasoning ability exhibited increased glucose metabolic rates in the face of increasing reasoning demands, whereas individuals with low reasoning ability did not show such increases (Larson, Haier, LaCasse, \& Hazen, 1995). Similarly, in an fMRI investigation of sentence comprehension, skilled readers showed larger neural adaptability as a function of word frequency than did less-skilled readers (Prat et al., 2007). Specifically, skilled readers activated the left hemisphere language regions more when reading sentences with low-frequency words than they did when reading sentences with highfrequency words, whereas less-skilled readers did not show differential activation in the two conditions. In other words, skilled readers showed more adaptive modulation of neural resources in the face of changing lexical demands than did less-skilled readers.

Neural adaptability is not limited to changes in the amount of activation. Research has shown that changes in cortical synchronization, or functional connectivity, as a function of task demand is another useful index of cortical adaptation. More skilled readers showed greater modulation of synchronization (increased functional connectivity) as a function of increased demands imposed by syntactic complexity and lexical (in)frequency. As either syntactic complexity or lexical (in) frequency demands increased, cortical synchronization of high-capacity readers increased, whereas synchronization of low-capacity readers tended to remain constant or decrease. In general, higher skill level is associated with greater modulation of cortical activation and synchronization as a function of task demands.

\section{Proposed Brain and Behavior Links}

Below, we provide a speculative account of how individual differences in brain attributes may be related to individual differences in speed of processing and fluid intelligence.

Speed of Processing. Researchers have repeatedly proposed that the primary source of variation in individuals' abilities to perform mental processes is the speed with which they can process information (e.g. Jensen, 1993; Vernon \& Jensen, 1983). The speed of human information processing is likely to vary as a function of the quality of the white matter tracts (constituting approximately $40 \%$ of brain volume) that provide the interconnectivity among cortical centers. Myelination (the basis of the whiteness of white matter) increases signal transmission speed by a factor of approximately 10. The amount of white matter (as indexed by volumetric measures) and quality or integrity of the white matter tracts (as indexed by DTI 
measures such as fractional anisotropy) should be a determinant of the speed of inter-center communication which is proposed to underpin higher-level thinking. Recent brain imaging capabilities have made it possible to examine the relation between the properties of white-matter tracts and cognitive function. For example, white matter continues to develop through youngadulthood (e.g., Schmithorst, Wilke, Dardzinski, \& Holland, 2001), and the degree of whitematter organization at any given age is positively correlated with IQ, especially in tracts connecting to the frontal lobe (Schmithorst, Wilke, Dardzinski, \& Holland, 2005). Furthermore, high-IQ adults have greater white matter volumes in a number of brain regions including the corpus callosum (Luders, 2007) and the regions connecting to Wernicke's Area (Haier, 2004). Recently, diffusion tensor imaging experiments have shown correlations between the integrity of white-matter tracts and specific skills. For example, children with better reading skill have higher left temporo-parietal white-matter tract integrity than do poor readers (Beaulieu et al, 2005). These findings collectively indicate that individual differences in the speed of processing in cognitive tasks may be underpinned to a large degree by the quality of white-matter tracts.

Fluid Intelligence. Our view of cognition as a network function leads to a new perspective of fluid intelligence. According to the new perspective, fluid intelligence corresponds to the ability to dynamically configure a collaborative information processing network of cortical centers to effectively deal with a novel cognitive challenge of some complexity. In this view, fluid intelligence does not reside in a particular place in the brain, but in the system's ability to bring together capabilities that are distributed across different parts of the brain. We propose that individuals systematically differ in this ability and that it underpins fluid intelligence.

An alternative view is that the frontal lobes are the seat of individual differences in fluid intelligence. To be sure, almost any test of fluid intelligence will draw on the frontal lobes, as studies have shown (Duncan et al., 2000; Grey, Chabris, \& Braver, 2003; Prabhakaran, Smith, Desmond, Glover, \& Gabrieli, 1997). In this alternative view, fluid intelligence is not a property of a cortical network, but resides in the flexibility (fluidity) of the algorithms that can be generated and executed by frontal lobe executive processes.

There is a third class of alternatives that are a hybrid of the first two, proposing that intelligence is related to the quality of the communication between the frontal lobes and other important brain regions. The Parietal-Frontal Integration Theory (P-FIT) proposes that intelligent cognitive functioning requires information synthesis between frontal and parietal regions (Jung \& Haier, 2007), thus singling out this particular pair of regions. A variant of P-FIT is that it is a more general ability of the frontal lobes to coordinate processes with posterior regions, including posterior temporal and occipital areas, that underpins intelligent functioning (Prabhakaran and Rypma, 2007). Although it is uncertain which of the three alternatives is preferable, it is certain that considerations of brain function have provided extremely interesting new hypotheses about the nature of human intelligence.

\section{Summary}

Looking to a complex system like the human brain for sources of individual differences leads to several new insights. First is our theme that characterizations of the neural basis of individual differences in cognition must include system-level attributes of the brain. Second is the theme that a complex system like the brain is likely to have multiple attributes that form the bases of individual differences in cognition. The brain is a magnificent biological system for thinking, and the systematic differences in thinking ability are surely reflections at least in part of the underlying biology. This truism can come to life if we consider how the new insights might influence our attitudes towards such issues as cognitive effects of normal aging, academic instruction, and recovery from various types of brain damage.

${ }^{1}$ There are multiple mechanisms that may account for an individual's high performance level on a cognitive task (e.g., prior practice in the task and/or inherent aptitude for the task); we will ignore such differences and focus on the neural underpinnings of the resulting performance level, under the tentative assumption that the different mechanisms yield relatively similar outcomes.

${ }^{2}$ We limit our discussion to individual differences in neurotypical populations, because the principles discussed here may differ in special populations.

References

Beaulieu, C., Plewes, C., Paulson, L. A., Roy, D., Snook, L., Concha, L., \& Phillips, L. (2004). Imaging brain connectivity in children with diverse reading ability. Neurolmage, 25, 1266-1271.

Boivin, M. J., Giordani, B., Berent, S., Amato, D. A., Lehtinen, S., Koeppe, R.A., Buchtel, H. A., Foster, N. L., \& Kuhl, D. E. (1992). Verbal fluency and positron emission tomographic mapping of regional cerebral glucose metabolism. Cortex, 28, 231-239.

Buchel, C., Coull, J. T., \& Friston, K. J. (1999). The predictive value of changes in effective connectivity for human learning. Science, 283, 1538-1541.

Friston, K. J., Jezzard, P., \& Turner, R. (1994). Analysis of functional MRI time-series. Human Brain Mapping, 1, 153-171.

Gray, J. R., Chabris, C. F., \& Braver, T. S. (2003). Neural mechanisms of general fluid intelligence. Nature Neuroscience, 6, 316-322.

Haier, R. J., Jung, R. E., Yeo, R. A., Head, K., \& Alkire, M. T. (2004). Structural brain variation and general 
intelligence. Neurolmage, 23, 425-433.

Haier, R. J., Siegel, B.V., Nuechterlein, K. H., Hazlett, E., Wu, J., Paek, J., Browning, H., \& Buchsbaum, M. S. (1988). Cortical glucose metabolic rate correlates of abstract reasoning and attention studied with positron emission tomography. Intelligence, 12, 199-217.

Jensen, A. R. (1993). Why is reaction time correlated with psychometric g? Psychological Science, 2, 53-56.

Jung, R. E. \& Haier, R. J. (2007). The parieto-frontal integration theory (P-FIT) of intelligence: Converging neuroimaging evidence. Behavioral and Brain Sciences, 30, 135-154.

Just, M.A. \& Varma, S. (2007). The organization of thinking: What functional brain imaging reveals about the neuroarchitecture of complex cognition. Cognitive, Affective, and Behavioral Neuroscience, 7, 153-191.

Larson, G. E., Haier, R. J., LaCasse, L., \& Hazen, K. (1955). Evaluation of a "mental effort" hypothesis for correlations between cortical metabolism and intelligence. Intelligence, 21, 267-278.

Luders, E., Narr, K. L., Bilder, R. M., Thompson, P. M., Szeszko, P. R., Hamilton, L., \& Toga, A.W. (2007). Positive correlations between corpus callosum thickness and intelligence. Neurolmage, 37, 1457-1464.

Maxwell, A. E., Fenwick, P. B., Fenton, G. W., \& Dollimore, J. (1974). Reading ability and brain function: A simple statistical model. Psychological Medicine, 4, 274-280.

Newman, S. D., Carpenter, P. A., Varma, S., \& Just, M. A. (2003). Frontal and parietal participation in problem solving in the Tower of London: fMRI and computational modeling of planning and high-level perception. Neuropsychologia, 41, 1668-1682.

Newman, S. D. \& Just, M. A. (2005). Cognition and Intelligence: Identifying the mechanisms of the Mind. In Robert J. Sternberg \& Jean Pretz (Eds.), Cognition and intelligence: Identifying the mechanisms of the mind (pp. 88-103). New York: Cambridge University Press.

Newman, S. D., Just, M. A., \& Carpenter, P. A. (2002). The synchronization of the human cortical working memory network. Neurolmage, $15,810-822$

Otsuka, Y., \& Osaka, N. (2005). An fMRI study of verbal working memory: Effects of aging on ACC-PFC network connectivity. Japanese Journal of Physiological Psychology and Psychophysiology, 23, 11-18.

Prabhakaran, V. \& Rypma, B. (2007). P-Fit and the neuroscience of intelligence: How well does P fit? Behavioral and Brain Sciences, 30, 166-167.

Prabhakaran, V., Smith, J. A. L., Desmond, J. E., Glover, G. H., \& Gabrieli, J. D. E. (1997). Neural substrates of fluid reasoning: An fMRI study of neocortical activation during performance of the Raven's Progressive Matrices Test. Cognitive Psychology, 33, 43-63.

Prat, C. S., Keller, T. A., \& Just, M. A. (2007). Individual differences in sentence comprehension: A functional magnetic resonance imaging investigation of syntactic and lexical processing demands. Journal of Cognitive Neuroscience, 19, 1950-1963.

Prat, C. S., Mason, R. A., \& Just, M. A. (in preparation). Individual differences in the role of the right hemisphere in causal inference comprehension.

Reichle, E. D., Carpenter, P. A., \& Just, M. A. (2000). The neural bases of strategy and skill in sentence-Picture Verification. Cognitive Psychology, 40, 261-295

Schmithorst, V. J., Wilke, M., Dardzinski, B. J., \& Holland, S. K. (2001). Correlation of white matter diffusivity and anisotropy with age during childhood and adolescence: A cross-sectional diffusion-tensor MR imaging study. Radiology, 222, 212-218.

Schmithorst, V. J., Wilke, M., Dardzinski, B. J., \& Holland, S. K. (2005). Cognitive functions correlate with white architecture in a normal pediatric population: A diffusion tensor MRI study. Human Brain Mapping, 26, 139-147.

Vernon, P. A. \& Jensen, A. R. (1983). Individual and group differences in intelligence and speed of information processing. Personality and Individual Differences, 5, 411-423.

(c) 2008 American Psychöogical Association

Science Directorate

750 First Street, NE • Washington, DC • 20002-4242

Phone: 202-336-6000 - TDD/TTY: 202-336-6123

Fax: 202-336-5953 - E-mail

PsychNET® | Terms of Use I Privacy Policy I Security I Advertise with us 